\title{
NOTES ON FOURIER ANALYSIS. XXX ON THE ABSOLUTE CONVERGENCE OF CERTAIN SERIES OF FUNCTIONS ${ }^{1}$
}

\author{
GEN-ICHIRÔ SUNOUCHI AND SHIGEKI YANO
}

1. Introduction. Recently O. SzÁsz $[4]^{2}$ has proved the following theorems which are generalizations of Fatou's theorem (cf. Zygmund $[5$, p. 132]).

ThEOREM A. If the series

$$
\left.\sum_{n=1}^{\infty} a_{n} \cos n x,\left|a_{n+1}\right| \leqq c\left|a_{n}\right|(c>0) ; n=1,2, \cdots\right),
$$

is absolutely convergent at a point $x_{0}$, then $\sum_{n=1}^{\infty}\left|a_{n}\right|<\infty$. The same is true for the series $\sum_{n=1}^{\infty} a_{n} \sin n x$, provided that $x_{0} \neq \equiv 0(\bmod \pi)$.

Theorem B. Let $\phi(x)$ be Riemann integrable in the interval $(0,1)$ and periodic with period 1 and $\int_{0}^{1}|\phi(x)| d x \neq 0$. If the series

$$
\sum_{n=1}^{\infty} a_{n} \phi(n x), 0<\left|a_{n+1}\right|<(1+c / n)\left|a_{n}\right|(c>0 ; n=1,2, \cdots),
$$

is absolutely convergent at an irrational point $x_{0}$, then $\sum_{n=1}^{\infty}\left|a_{n}\right|<\infty$.

There are some gaps between the conditions for the sequences of coefficients $\left\{a_{n}\right\}$ in these two theorems. In $\$ 2$, we shall prove that the conditions for $\left\{a_{n}\right\}$ in Theorem $\mathrm{B}$ can be replaced by those in Theorem A.

On the other hand Reves and Szász [2] have proved the analogues of Cantor-Lebesgue's theorem and Denjoy-Lusin's theorem for the double trigonometric series. In $\S 3$, we shall generalize these theorems: the former is on the line of Mazur-Orlicz' generalization for CantorLebesgue's theorem [1, Theorem 1 and its corollary] and the latter is in the direction of Salem's generalization for Denjoy-Lusin's theorem [3, Theorem X].

2. Theorems of Fatou-Szász type. We shall give here the generalizations of Theorems A and B.

Theorem 1. Let $\phi(x)$ be defined in the interval $(0,1)$ and periodic with period 1 and suppose that there exists an interval $I=(a, b)$,

Received by the editors January 9, 1950 and, in revised form, July 10, 1950.

${ }_{1}$ The authors express their hearty thanks to the referee who gave many valuable suggestions, especially that the original proof of Theorem 1 was incomplete.

2 Numbers in brackets refer to the literature at the end of the paper. 
$0 \leqq a<b \leqq 1$, on which $\phi(x)$ is greater than a positive constant $d$. If the series $\sum_{n=1}^{\infty} a_{n} \phi(n x)\left(a_{n} \geqq 0\right)$ is absolutely convergent at an irrational point $\xi$ and if $0<a_{n+1} \leqq c a_{n}(c>0 ; n=1,2, \cdots)$, then $\sum_{n=1}^{\infty} a_{n}<\infty$.

For the proof we need the following lemma.

LEMмA 1. Let $J$ be an interval and $\theta$ be an irrational number contained in the interval $(0,1)$. Then for an arbitrary real number $\alpha$ there exists a positive integer $N$ independent of $\alpha$ such that at least one of the numbers $\alpha+n \theta, n=1,2, \cdots, N$, is contained in the interval $J$ with modulus 1.

Proof. We shall prove more precisely that if we denote the number of the points in the sequence $\{\alpha+\nu \theta\}, \nu=1,2, \cdots, n$, which are contained with modulus 1 in the interval $J$ by $n_{J}(\alpha)$, then the ratio $n_{J}(\alpha) / n$ tends independently on $\alpha$ to the length of the interval $J$ as $n \rightarrow \infty$; that is, for any given $\epsilon>0$, there is a positive integer $N$ independent of $\alpha$ such that $\left|n_{J}(\alpha) / n-l\right|<\epsilon$ for $n>N$, where $l$ is the length of the interval $J$.

To prove this, let $f(x)$ be the function which is obtained by extending the characteristic function of the interval $J$ with period 1 . Then there exist two trigonometrical polynomials $p(x)$ and $P(x)$ which satisfy the inequalities

$$
\begin{aligned}
p(x) \leqq f(x) \leqq P(x) & \text { in }(0,1), \\
\int_{0}^{1}[f(x)-p(x)] d x<\epsilon / 2, & \int_{0}^{1}[P(x)-f(x)] d x<\epsilon / 2 .
\end{aligned}
$$

We can write

$$
p(x)=\sum_{k=-m}^{m} c_{k} e^{2 \pi i k x}, \quad P(x)=\sum_{k=-m}^{m} C_{k} e^{2 \pi i b x} .
$$

Remembering that

$$
\frac{n_{J}(\alpha)}{n}=\frac{1}{n} \sum_{\nu=1}^{n} f(\alpha+\nu \theta)
$$

we have

$$
\frac{1}{n} \sum_{\nu=1}^{n} p(\alpha+\nu \theta) \leqq \frac{n_{J}(\alpha)}{n}
$$

$$
\leqq \frac{1}{n} \sum_{\nu=1}^{n} P(\alpha+\nu \theta)
$$


by (1).

On the other hand

$$
\begin{aligned}
\frac{1}{n} \sum_{\nu=1}^{n} p(\alpha+\nu \theta) & =\frac{1}{n} \sum_{\nu=1}^{n} \sum_{k=-m}^{m} c_{k} e^{2 \pi i k(\alpha+\nu \theta)} \\
& =\frac{1}{n} \sum_{\nu=1}^{n} \sum_{k=-m}^{m} c_{k} e^{2 \pi i k \alpha} e^{2 \pi i k \nu \theta} \\
& =c_{0}+\frac{1}{n} \sum_{k=-m}^{m} c_{k} e^{2 \pi i k \alpha} \sum_{v=1}^{n} e^{2 \pi i k v \theta} \\
& =c_{0}+\frac{1}{n} \sum_{k=-m}^{\prime} c_{k} e^{2 \pi i k \alpha} \frac{e^{2 \pi i k \theta}-e^{2 \pi i k(n+1) \theta}}{1-e^{2 \pi i k \theta}},
\end{aligned}
$$

where $\sum^{\prime}$ denotes the summation omitting the term for $k=0$. Thus we have

$$
\begin{aligned}
\mid \int_{0}^{1} p(x) d x-\frac{1}{n} \sum_{n=1}^{n} p(\alpha & +\nu \theta) \mid \\
& \leqq \frac{1}{n}\left|\sum_{k=-m}^{m} c_{k} e^{2 \pi i k \alpha} \frac{e^{2 \pi i k \theta}-e^{2 \pi i k(n+1) \theta}}{1-e^{2 \pi i k \theta}}\right| \\
& \leqq \frac{1}{n} \sum_{k=-m}^{m}\left|c_{k}\right| \frac{2}{\left|1-e^{2 \pi i k \theta}\right|} \\
& \leqq \frac{2 \Delta}{n} \sum_{k=-m}^{m}\left|c_{k}\right|,
\end{aligned}
$$

where

$$
\Delta=\max _{-m \leqq k \leqq m, k \neq 0} \frac{1}{\left|1-e^{2 \pi i k \theta}\right|}
$$

and $\Delta$ is finite since $\theta$ is irrational and $m$ is fixed; moreover it is evident from the definition that $\Delta$ is independent of $\alpha$.

Therefore if we choose $N_{1}$ such that

$$
N_{1}>4 \Delta \sum_{k=-m}^{m}\left|c_{k}\right| / \epsilon
$$

we have

$$
\left|\int_{0}^{1} p(x) d x-\frac{1}{n} \sum_{n=1}^{n} p(\alpha+\nu \theta)\right|<\epsilon / 2 \quad \text { for } n>N_{1} .
$$

Quite similarly we can choose $N_{2}$ such that 


$$
\left|\int_{0}^{1} P(x) d x-\frac{1}{n} \sum_{\nu=1}^{n} P(\alpha+\nu \theta)\right|<\epsilon / 2 \quad \text { for } n>N_{2} .
$$

Let us take $N=\max \left(N_{1}, N_{2}\right)$, then we have

$$
\begin{aligned}
\int_{0}^{1} p(x) d x-\epsilon / 2 & <\frac{1}{n} \sum_{\nu=1}^{n} p(\alpha+\nu \theta) \leqq \frac{n_{J}(\alpha)}{n} \\
& \leqq \frac{1}{n} \sum_{n=1}^{n} P(\alpha+\nu \theta)<\int_{0}^{1} P(x) d x+\epsilon / 2
\end{aligned}
$$

from (5), (10), and (11), and finally

$$
\int_{0}^{1} f(x) d x-\epsilon \leqq \frac{n_{J}(\alpha)}{n} \leqq \int_{0}^{1} f(x) d x+\epsilon
$$

from (2) and (12). Since $\int_{0}^{1} f(x) d x=l$ is the length of the interval $J$, this proves our proposition.

We shall proceed to the proof of Theorem 1. Using Lemma 1, we can choose a positive integer $k$ such that for any integer $n$ at least one of the $k$ numbers $n \xi+\xi, n \xi+2 \xi, \cdots, n \xi+k \xi$ is contained with modulus 1 in the interval $I$. From the assumption on $a_{n}$ (since we can suppose $c>1$ without any loss of generality) we have

$$
a_{k-m} \geqq a_{k} / c^{m} \geqq a_{k} / c^{k} \quad(m \leqq k) .
$$

Then

$$
\begin{aligned}
\sum_{n=1}^{\infty} a_{n}|\phi(n \xi)| & =\sum_{n=0}^{\infty} \sum_{m=1}^{k} a_{n k+m}|\phi((n k+m) \xi)| \\
& \geqq\left(d / c^{k}\right) \sum_{n=1}^{\infty} a_{n k} .
\end{aligned}
$$

Consequently

$$
\begin{aligned}
\sum_{n=1}^{\infty} a_{n} & =\sum_{n=1}^{\infty} \sum_{m=1}^{k} a_{n k+m} \leqq \sum_{n=1}^{\infty} \sum_{m=1}^{k} a_{n k} c^{m} \\
& \leqq \frac{c^{k+1}-1}{c-1} \sum_{n=1}^{\infty} a_{n k} \leqq \frac{c^{k}\left(c^{k+1}-1\right)}{d(c-1)} \sum_{n=1}^{\infty} a_{n}|\phi(n \xi)|<\infty .
\end{aligned}
$$

This proves the theorem.

REMARK. This theorem implies Theorem B in $\$ 1$; in fact if $\phi(x)$ is Riemann integrable, $\phi(x)$ is continuous almost everywhere, therefor the assumption $\int_{0}^{1}|\phi(x)| d x \neq 0$ assures the existence of an interval on which $|\phi(x)|$ is greater than a positive constant. 
THEOREM 2. Besides the assumption on $\phi(x)$ and $\left\{a_{n}\right\}$ in Theorem 1 , we assume that $\phi(0) \neq 0$; then the absolute convergence of the series $\sum_{n=1}^{\infty} a_{n} \phi(n x)$ for any real $x_{0}$ implies the convergence of the series $\sum_{n=1}^{\infty} a_{n}$.

Proof. If $x_{0}$ is irrational, then the result follows from Theorem 1. In case $x_{0}$ is rational, say $x_{0}=p / q$ where $p$ and $q$ are integers, the numbers $n q x_{0}(n=1,2, \cdots)$ are all equal to 0 with modulus 1 . An argument quite similar to that in the proof of Theorem 1 proves also the required result.

This theorem implies Theorem A in $\$ 1$ for the case of cosine series. Corresponding to the case of sine series we shall prove the following theorem.

THEOREM 3. Let $\phi(x)$ and $a_{n}$ satisfy the same conditions as in Theorem 1. If the series $\sum_{n=1}^{\infty} a_{n} \phi(n x)$ converges absolutely at an irrational $x_{0}$ or at a rational $x_{0}, x_{0}=p / q$ say, where $p$ and $q$ are relatively prime integers and there exists at least one of the numbers $r / q$ $(r=1, \cdots, q-1)$ which is not the zero of $\phi(x)$, then the series $\sum_{n=1}^{\infty} a_{n}$ converges.

Proof. For irrational $x_{0}$, this reduces to Theorem 1. Let us suppose that $\phi\left(r_{0} / q\right) \neq 0,0<r_{0} \leqq q-1$. Since $p$ and $q$ are relatively prime, there are two integers $u$ and $v$ such that $u p+v q=1$, that is, $u p / q$ $=1 / q-v$. Consequently there is a number in the $u r_{0}$ consecutive numbers of the sequence $\left\{n x_{0}\right\}$ which is equal to $r_{0} / q$ with modulus 1 . Therefore the same argument as in the proof of Theorem 1 gives the required result.

3. Double trigonometrical series. We shall first state the following lemmas which are generalizations of the results of Mazur-Orlicz [1].

Lemma 2. If $f(x)$ is measurable, bounded, and periodic, we have for every integrable function $g(x)$ in $(a, b)$

$$
\lim _{m, n \rightarrow \infty} \int_{a}^{b} f\left(\omega_{m n} x+\theta_{m n}\right) g(x) d x=\mathfrak{M}(f) \int_{a}^{b} g(x) d x,
$$

where $\mathfrak{M}(f)=\int_{0}^{l} f(x) d x / l, l$ being the period of $f(x)$, and $\left\{\omega_{m n}\right\}$ and $\left\{\theta_{m n}\right\}$ are any sequences of real numbers such that lim $\omega_{m n}=+\infty$.

ProOF. If $m_{i}, n_{i} \rightarrow \infty(i \rightarrow \infty)$, we have by a result of Mazur and Orlicz [1, Lemma 1]

$$
\lim _{i \rightarrow \infty} \int_{a}^{b} f\left(\omega_{m_{i} n_{i}} x+\theta_{m_{i} n_{i}}\right) g(x) d x=\mathfrak{M}(f) \int_{a}^{b} g(x) d x .
$$


This proves Lemma 2.

Lemмa 3. If $f(x)$ is measurable and periodic, then we have almost everywhere,

$$
\limsup _{m, n \rightarrow \infty}\left|a_{m n} f\left(\omega_{m n} x+\theta_{m n}\right)\right|=\limsup _{m, n \rightarrow \infty}\left|a_{m n}\right| \text { ess sup }|f(x)|,
$$

where $\left\{\omega_{m n}\right\},\left\{\theta_{m n}\right\}$ are the same as in Lemma 2 and $\left\{a_{m n}\right\}$ is any sequence of real numbers.

Proof. We can choose a sequence of suffixes $\left\{m_{i}, n_{i}\right\}$ such that $m_{i}, n_{i} \rightarrow \infty(i \rightarrow \infty)$ and $\lim _{i \rightarrow \infty}\left|a_{m_{i} n_{i}}\right|=\lim \sup _{m, n \rightarrow \infty}\left|a_{m n}\right|$. Then by Mazur and Orlicz [1, Theorem 1] we have almost everywhere

$$
\begin{aligned}
\limsup _{i \rightarrow \infty}\left|a_{m_{i} n_{i}} f\left(\omega_{m_{i} n_{i}} x+\theta_{m_{i} n_{i}}\right)\right| & =\limsup _{i \rightarrow \infty}\left|a_{m_{i} n_{i}}\right| \text { ess sup }|f(x)| \\
& =\limsup _{m, n \rightarrow \infty}\left|a_{m n}\right| \text { ess sup }|f(x)| .
\end{aligned}
$$

It follows that almost everywhere

$$
\limsup _{m, n \rightarrow \infty}\left|a_{m n} f\left(\omega_{m n} x+\theta_{m n}\right)\right| \geqq \limsup _{m, n \rightarrow \infty}\left|a_{m n}\right| \text { ess sup }|f(x)| \text {. }
$$

Since the inverse inequality is evident, our lemma is proved.

Lemma 4. If $f(x)$ and $g(x)$ are linearly independent, bounded, measurable, and periodic with the same period, then for any sequences $\left\{a_{m n}\right\}$ and $\left\{b_{m n}\right\}$, we have almost everywhere

$$
\begin{aligned}
\limsup _{m, n \rightarrow \infty} \mid a_{m n} f\left(\omega_{m n} x+\theta_{m n}\right)+b_{m n} g\left(\omega_{m n} x\right. & \left.+\theta_{m n}\right) \mid \\
& \geqq c \limsup _{m, n \rightarrow \infty}\left(\left|a_{m n}\right|+\left|b_{m n}\right|\right),
\end{aligned}
$$

where $c=\inf _{|a|+|b|=1}\{$ ess sup $|a f(x)+b g(x)|\}>0$. (See Mazur-Orlicz [1, Theorem 3 and its corollary].)

Proof. We can choose a sequence of indices $m_{i}, n_{i}\left(m_{i}, n_{i} \rightarrow \infty\right.$ as $i \rightarrow \infty)$ such that $\lim \sup _{m, n \rightarrow \infty}\left(\left|a_{m n}\right|+\left|b_{m n}\right|\right)=\lim _{i \rightarrow \infty}\left(\left|a_{m_{i} n_{i}}\right|\right.$ $\left.+\left|b_{m_{i} n_{i}}\right|\right)$. Then we have almost everywhere

$$
\begin{array}{rl}
\limsup _{m, n \rightarrow \infty} \mid a_{m n} & f\left(\omega_{m n} x+\theta_{m n}\right)+b_{m n} g\left(\omega_{m n} x+\theta_{m n}\right) \mid \\
& \geqq \limsup _{i \rightarrow \infty}\left|a_{m_{i} n_{i}} f\left(\omega_{m_{i} n_{i}} x+\theta_{m_{i} n_{i}}\right)+b_{m_{i} n_{i}} g\left(\omega_{m_{i} n_{i}} x+\theta_{m_{i} n_{i}}\right)\right| \\
& \geqq c \limsup _{i \rightarrow \infty}\left(\left|a_{m_{i} n_{i}}\right|+\left|b_{m_{i} n_{i}}\right|\right) \\
& =c \lim _{m, n \rightarrow \infty} \sup _{i}\left(\left|a_{m n}\right|+\left|b_{m n}\right|\right),
\end{array}
$$


by a result of Mazur and Orlicz [1, Theorem 3 and its corollary]. This proves the lemma.

We shall now prove the following theorem which is a generalization of Cantor-Lebesgue's theorem for double trigonometric series.

THEOREM 4. Let $f(x), g(x)$ be any measurable, periodic functions, and $\phi(y), \psi(y)$ be linearly independent, bounded, measurable, and periodic functions with the same period, and let $\left\{\lambda_{m n}\right\},\left\{\omega_{m n}\right\}$, and $\left\{\omega_{m n}^{\prime}\right\}$ be the sequences which tend to $\infty$ as $m, n \rightarrow \infty$, and $\left\{\tau_{m n}\right\},\left\{\theta_{m n}\right\},\left\{\theta_{m n}^{\prime}\right\}$ be any sequences. Then for any sequences $\left\{p_{m n}\right\}$ and $\left\{q_{m n}\right\}$, there exists a constant $c>0$ depending only on $\phi$ and $\psi$, such that

$$
\begin{aligned}
& \limsup _{m, n \rightarrow \infty} \mid p_{m n} f\left(\omega_{m n} x+\theta_{m n}\right) \phi\left(\lambda_{m n} y+\tau_{m n}\right) \\
& +q_{m n} g\left(\omega_{m n}^{\prime} x+\theta_{m n}^{\prime}\right) \psi\left(\lambda_{m n} y+\tau_{m n}\right) \mid \\
& \geqq c \max \left\{\limsup _{m, n \rightarrow \infty}\left|p_{m n}\right| \operatorname{ess} \sup |f(x)|\right. \text {, } \\
& \left.\lim \sup \left|q_{m n}\right| \operatorname{ess} \sup |g(x)|\right\}
\end{aligned}
$$

for almost every $(x, y)$.

Proof. If we fix an $x$, we have

$$
\begin{aligned}
\limsup _{m, n \rightarrow \infty} \mid p_{m n} f\left(\omega_{m n} x+\theta_{m n}\right) \phi\left(\lambda_{m n} y+\tau_{m n}\right) & \\
& \quad+q_{m n} g\left(\omega_{m n}^{\prime} x+\theta_{m n}^{\prime}\right) \psi\left(\lambda_{m n} y+\tau_{m n}\right) \mid \\
\geqq & c \limsup _{m, n \rightarrow \infty}\left\{\left|p_{m n} f\left(\omega_{m n} x+\theta_{m n}\right)\right|+\left|q_{m n} g\left(\omega_{m n}^{\prime} x+\theta_{m n}^{\prime}\right)\right|\right\} \\
\geqq & c \max \left\{\limsup _{m, n \rightarrow \infty}\left|p_{m n} f\left(\omega_{m n} x+\theta_{m n}\right)\right|,\right. \\
& \left.\limsup _{m, n \rightarrow \infty}\left|q_{m n} g\left(\omega_{m n}^{\prime} x+\theta_{m n}^{\prime}\right)\right|\right\}
\end{aligned}
$$

by Lemma 4. Applying Lemma 3, we have for almost every $x$

$$
\limsup _{m, n \rightarrow \infty}\left|p_{m n} f\left(\omega_{m n} x+\theta_{m n}\right)\right|=\limsup _{m, n \rightarrow \infty}\left|p_{m n}\right| \text { ess sup }|f(x)|
$$

and

$$
\limsup _{m, n \rightarrow \infty}\left|q_{m n} g\left(\omega_{m n}^{\prime} x+\theta_{m n}^{\prime}\right)\right|=\limsup _{m, n \rightarrow \infty}\left|q_{m n}\right| \text { ess sup }|g(x)| .
$$

Then by Fubini's theorem we get the theorem.

Corollary. Let 


$$
\begin{aligned}
A_{m n}(x, y)= & a_{m n} \cos m x \cos n y+b_{m n} \sin m x \sin n y \\
& +c_{m n} \cos m x \sin n y+d_{m n} \sin m x \sin n y .
\end{aligned}
$$

If $A_{m n}(x, y)$ tends to zero as $m, n \rightarrow \infty$, for every $(x, y)$ belonging to a plane set of positive measure, then

$$
\rho_{m n}=\left(a_{m n}^{2}+b_{m n}^{2}+c_{m n}^{2}+d_{m n}^{2}\right)^{1 / 2} \rightarrow 0 \quad \text { as } m, n \rightarrow \infty .
$$

This is the Reves-Szász generalization of the Cantor-Lebesgue theorem [1, Theorem 1].

Proof. Let us put in Theorem 4

$$
\begin{gathered}
f(x)=g(x)=\cos x, \quad \phi(y)=\cos y, \quad \psi(y)=\sin y, \\
p_{m n}^{2}=a_{m n}^{2}+b_{m n}^{2}, \quad q_{m n}^{2}=c_{m n}^{2}+d_{m n}^{2} .
\end{gathered}
$$

Then we may write (see Reves and Szász [2, Theorem 1])

$$
A_{m n}(x, y)=p_{m n} \cos \left(m x-\theta_{m n}\right) \cos n y+q_{m n} \cos \left(m x-\theta_{m n}^{\prime}\right) \sin n y .
$$

Therefore we get the corollary by Theorem 4 .

TheOREM 5. Let $A_{m n}(x, y)$ and $\rho_{m n}$ be the same as in the above corollary. Then the plane set $E,^{3}$

$$
E=\left\{(x, y)\left|\limsup _{m, n \rightarrow \infty} \sum_{i, j=1}^{m, n}\right| A_{i j}(x, y) \mid / \sum_{i, j=1}^{m, n} \rho_{i j}<\alpha\right\}
$$

is of measure zero, provided that $\alpha<4 / 2^{1 / 2} \pi^{2}$ and $\sum_{m, n=1}^{\infty} \rho_{m n}=\infty$.

Proof. Let $f(x, y)$ be the characteristic function of the set $E$, then we have

$$
\begin{aligned}
\alpha f(x, y) & \geqq \limsup _{m, n \rightarrow \infty} f(x, y) \sum_{i, j=1}^{m, n}\left|A_{i j}(x, y)\right| / \sum_{i, j=1}^{m, n} \rho_{i j} \\
& =\limsup _{m, n \rightarrow \infty} \frac{f(x, y) \sum_{i, j=1}^{m, n}\left|A_{i j}(x, y)\right|}{\sum_{i, j=1}^{m, n}\left(\Delta_{i j}(x)\right)^{1 / 2}} \frac{\sum_{i, j=1}^{m, n}\left(\Delta_{i j}(x)\right)^{1 / 2}}{\sum_{i, j=1}^{m, n} \rho_{i j}} \\
& \geqq \limsup _{m, n \rightarrow \infty} \frac{1}{2^{1 / 2}} \frac{f(x, y) \sum_{i, j=1}^{m, n}\left|A_{i j}(x, y)\right|}{\sum_{i, j=1}^{m, n}\left(\Delta_{i j}(x)\right)^{1 / 2}} \\
& \frac{\sum_{i, j=1}^{m, n}\left\{\left|p_{i j} \cos \left(i x-\theta_{i j}\right)\right|+\left|q_{i j} \cos \left(i x-\theta_{i j}^{\prime}\right)\right|\right\}}{\sum_{i, j=1}^{m, n} \rho_{i j}}
\end{aligned}
$$

${ }^{3}$ Of course, we suppose that $E$ is contained in the square $(0,2 \pi ; 0,2 \pi)$. 
where $\Delta_{i j}(x)=p_{i j}^{2} \cos ^{2}\left(i x-\theta_{i j}\right)+q_{i j}^{2} \cos ^{2}\left(i x-\theta_{i j}^{\prime}\right)$.

Applying Lebesgue's theorem we get

$$
\begin{gathered}
\alpha \int_{0}^{2 \pi} f(x, y) d y \geqq \frac{1}{2^{1 / 2}} \lim _{m, n \rightarrow \infty} \frac{\sum_{i, j=1}^{m, n} \int_{0}^{2 \pi} f(x, y)\left|A_{i j}(x, y)\right| d y}{\sum_{i, j=1}^{m, n}\left(\Delta_{i j}(x)\right)^{1 / 2}} \\
. \frac{\sum_{i, j=1}^{m, n}\left\{\left|p_{i j} \cos \left(i x-\theta_{i j}\right)\right|+\left|q_{i j} \cos \left(i x-\theta_{i j}^{\prime}\right)\right|\right\}}{\sum_{i, j=1}^{m, n} \rho_{i j}} .
\end{gathered}
$$

Since $\sum \rho_{m n}=\infty$, we can easily verify that

$$
\begin{aligned}
\sum_{i, j=1}^{\infty}\left(\Delta_{i j}(x)\right)^{1 / 2} \geqq \frac{1}{2^{1 / 2}} \sum_{i, j=1}^{\infty}\left\{\mid p_{i j} \cos (i x\right. & \left.-\theta_{i j}\right) \mid \\
& \left.+\left|q_{i j} \cos \left(i x-\theta_{i j}^{\prime}\right)\right|\right\}=\infty
\end{aligned}
$$

for almost every $x$. For such $x$, we have by Lemma 2

$$
\lim _{m, n \rightarrow \infty} \frac{\sum_{i, j=1}^{m, n} \int_{0}^{2 \pi} f(x, y)\left|A_{i j}(x, y)\right| d y}{\sum_{i, j=1}^{m, n}\left(\Delta_{i j}(x)\right)^{1 / 2}}=\frac{2}{\pi} \int_{0}^{2 \pi} f(x, y) d y .
$$

Hence

$$
\begin{aligned}
\alpha \int_{0}^{2 \pi} f(x, y) d y \geqq \frac{2}{2^{1 / 2} \pi} \int_{0}^{2 \pi} f(x, y) d y \\
\quad \cdot \limsup _{m, n \rightarrow \infty} \frac{\sum_{i, j=1}^{m, n}\left\{\left|p_{i j} \cos \left(i x-\theta_{i j}\right)\right|+\left|q_{i j} \cos \left(i x-\theta_{i j}^{\prime}\right)\right|\right\}}{\sum_{i, j=1}^{m, n} \rho_{i j}} .
\end{aligned}
$$

Since by Lemma 2

$$
\begin{aligned}
\lim _{i, j \rightarrow \infty} \int_{0}^{2 \pi} \int_{0}^{2 \pi} f(x, y)\left|\cos \left(i x-\theta_{i j}\right)\right| d x d y \\
=\frac{2}{\pi} \int_{0}^{2 \pi} \int_{0}^{2 \pi} f(x, y) d x d y,
\end{aligned}
$$

we have

$$
\alpha \int_{0}^{2 \pi} \int_{0}^{2 \pi} f(x, y) d x d y \geqq \frac{4}{2^{1 / 2} \pi^{2}} \int_{0}^{2 \pi} \int_{0}^{2 \pi} f(x, y) d x d y .
$$

If $\alpha<4 / 2^{1 / 2} \pi^{2}$, then we get

$$
\int_{0}^{2 \pi} \int_{0}^{2 \pi} f(x, y) d x d y=0
$$


that is, $E$ is of measure zero. Thus we get the theorem.

This is a generalization of a theorem of Salem [3, Theorem X]. Denjoy-Lusin's theorem for double trigonometrical series, which was given by Reves and Szász [2, Theorem 2], can be easily derived from Theorem 4.

\section{LITERATURE}

1. S. Mazur and W. Orlicz, Sur quelques proprietes de fonctions periodiques et presque-périodiques, Studia Mathematica vol. 8 (1940) pp. 1-15.

2. G. Reves and O. Szász, Some theorems on double trigonometrical series, Duke Math. J. vol. 9 (1942) pp. 693-705.

3. R. Salem, The absolute convergence of trigonometrical series, Duke Math. J. vol. 8 (1941) pp. 317-334.

4. O. Szász, On the absolute convergence of trigonometrical series, Ann. of Math. vol. 47 (1946) pp. 213-220.

5. A. Zygmund, Trigonometrical series, Warsaw, 1935.

TôHOKU UNIVERSITY 